\title{
Metabolic regulation of calcium pumps in pancreatic cancer: role of phosphofructokinase-fructose- bisphosphatase-3 (PFKFB3)
}

\author{
D. A. Richardson ${ }^{1}$, P. Sritangos ${ }^{1}$, A. D. James ${ }^{2}$, A. Sultan ${ }^{1}$ and J. I. E. Bruce ${ }^{1 *}$
}

\begin{abstract}
Background: High glycolytic rate is a hallmark of cancer (Warburg effect). Glycolytic ATP is required for fuelling plasma membrane calcium ATPases (PMCAs), responsible for extrusion of cytosolic calcium, in pancreatic ductal adenocarcinoma (PDAC). Phosphofructokinase-fructose-bisphosphatase-3 (PFKFB3) is a glycolytic driver that activates key rate-limiting enzyme Phosphofructokinase-1; we investigated whether PFKFB3 is required for PMCA function in PDAC cells.

Methods: PDAC cell-lines, MIA PaCa-2, BXPC-3, PANC1 and non-cancerous human pancreatic stellate cells (HPSCs) were used. Cell growth, death and metabolism were assessed using sulforhodamine-B/tetrazolium-based assays, poly-ADPribose-polymerase (PARP1) cleavage and seahorse XF analysis, respectively. ATP was measured using a luciferase-based assay, membrane proteins were isolated using a kit and intracellular calcium concentration and PMCA activity were measured using Fura-2 fluorescence imaging.
\end{abstract}

Results: PFKFB3 was highly expressed in PDAC cells but not HPSCs. In MIA PaCa-2, a pool of PFKFB3 was identified at the plasma membrane. PFKFB3 inhibitor, PFK15, caused reduced cell growth and PMCA activity, leading to calcium overload and apoptosis in PDAC cells. PFK15 reduced glycolysis but had no effect on steady-state ATP concentration in MIA PaCa-2.

Conclusions: PFKFB3 is important for maintaining PMCA function in PDAC, independently of cytosolic ATP levels and may be involved in providing a localised ATP supply at the plasma membrane.

Keywords: Metabolism, Glycolysis, PFKFB3, Calcium pumps, PMCA, Calcium overload, Pancreatic cancer, PDAC

\section{Introduction}

Pancreatic ductal adenocarcinoma (PDAC) is the most common form of pancreatic cancer and with a 5-year survival rate of $<3 \%$, it has one of the worst survival rates of all cancers $[1,2]$. Currently, the only curative treatment for PDAC is surgical resection, which is only possible for a fraction of patients and is often unsuccessful [3]. PDAC is often resistant to currently available treatments and there is a clear unmet need for novel drugs to specifically target PDAC.

PDAC cells undergo a metabolic switch from predominantly mitochondrial to highly glycolytic metabolism,

\footnotetext{
* Correspondence: jason.bruce@manchester.ac.uk

${ }^{1}$ Division of Cancer Sciences, School of Medical Sciences, University Of

Manchester, Michael Smith Building, Oxford Road, Manchester M13 9PT, UK

Full list of author information is available at the end of the article
}

referred to as the Warburg effect [4]. The Warburg phenotype facilitates numerous cancer hallmarks including rapid proliferation, invasion and immune evasion [5-8]. Our recent work has identified that glycolytic ATP is required to fuel ATP-dependent plasma membrane calcium ATPases (PMCAs), responsible for maintaining low intracellular calcium $\left(\left[\mathrm{Ca}^{2+}\right]_{\mathrm{i}}\right)$. Inhibition of glycolysis but not mitochondrial metabolism leads to attenuation of PMCA function, cytotoxic calcium overload and cell death in PDAC cells [9]. Moreover, reversal of the Warburg effect protects PMCA function in PDAC cells treated with glycolytic inhibitors [10]. Taken together, this suggests that the glycolytic dependency of PMCAs may present a novel therapeutic target in PDAC.

(c) The Author(s). 2020 Open Access This article is distributed under the terms of the Creative Commons Attribution 4.0 International License (http://creativecommons.org/licenses/by/4.0/), which permits unrestricted use, distribution, and 
After identifying that a glycolytic ATP supply to PMCAs is critical for PDAC cell survival, the next logical step was to identify key glycolytic enzymes that are upregulated in PDAC and contribute to this phenotype.

Pyruvate kinase M2 (PKM2) is a splice variant of the PKM gene that is expressed in some rapidly diving cells and overexpressed in many cancers, including PDAC, and has been described as a 'master regulator' of the Warburg effect [11-13]. PKM2 catalyses the final step in glycolysis which produces pyruvate and generates ATP. Though cancer cells exhibit a high rate of glycolysis, PKM2 has a lower catalytic activity than PKM1; this produces a bottleneck in glycolysis which allows the build-up of glycolytic intermediates that can be utilised for anabolic processes, at the expense of ATP production [14-17].

Phosphofructokinase-1 (PFK1) is responsible for converting fructose-6-phosphate (F6P) to fructose-1,6-bisphosphate (F16BP), which is the major rate-limiting reaction in glycolysis. PFK1 is overexpressed in numerous cancers and correlates with poor prognosis in lung cancer [18-22]. However, PFK1 is inhibited by a high ATP:AMP ratio. Cancer cells have been reported to have a low intracellular ATP concentration, thus preventing inhibition of PFK1 and driving the Warburg phenotype [19]. F16BP, the product of PFK1 activity also activates pyruvate kinase M2 (PKM2), responsible for the production of pyruvate, the final step of glycolysis, thus acting to drive glycolysis further [16].

PFK1 activity can also be increased by fructose-2,6bisphosphate (F26BP), a potent allosteric activator that drives glycolytic flux. F26BP is produced by phosphofructokinase-fructose-bisphosphatases (PFKFBs), which phosphorylate F6P to produce F26BP. PFKFBs are bifunctional enzymes that also possess phosphatase activity and can, therefore, remove phosphate from F26BP to regenerate F6P. There are four PFKFB isoforms (PFKFB1-4), of which PFKFB3 exhibits the highest kinase:phosphatase activity of all $(\sim 700$-fold). PFKFB3 is able to produce F26BP at a high rate and thus drives the Warburg effect $[23,24]$. Under hypoxic conditions, this kinase:phosphatase activity can be increased up to 3000-fold following phosphorylation at Ser460 by PKA or AMPK [25]. Hypoxia is a common feature of PDAC and hypoxia-inducible factor HIF1- $\alpha$ has been shown to induce expression of PFKFB3 [26].

PFKFB3 is overexpressed in numerous cancers including PDAC $[27,28]$; this finding led to the development of the specific small-molecule inhibitor $3 \mathrm{PO}$ and then more potent 3PO derivatives: PFK15 and PFK158 [24]. PFK15 has been effective in reducing tumour cell growth in vitro and has also reduced tumour growth and metastasis in xenograft models [29-31]. Importantly, neither animal studies nor phase I trials has identified any major detrimental effects of PFK158 suggesting that there are no off-target effects and that inhibition of PFKFB3 is not ubiquitously harmful [32-34].
The present study aimed to test whether the PFKFB3, a driver of the Warburg phenotype, plays a role in providing a glycolytic ATP supply to PMCAs in PDAC.

\section{Materials and methods Bioinformatics}

Oncomine software was used to generate heatmaps (Thermo Fisher Scientific, Ann Arbor, MI) and access expression data from the Badea Pancreas study [35]. Survival data was accessed and Kaplan-Meier plot generated using PROGgeneV2 (Indiana University Purdue University, Indianapolis, IN).

\section{Cell culture}

MIA PaCa-2 and PANC1 cells were obtained from the ATCC (Manassas, Virginia, USA), whereas human pancreatic stellate cells, isolated from resected tumour tissue (HPSCs) were a kind gift from Professor David Yule (University of Rochester, New York, USA). BxPC-3 cells were a kind gift from Dr. Ayse Latif (University of Manchester, UK). All cell types were grown in $75-\mathrm{cm}^{2}$ culture-treated flasks (Corning, New York, USA) in high-glucose Dulbecco's modified Eagle's media (DMEM) or RPMI-1640 (Sigma Aldrich, Gillingham, UK). All media was supplemented with $10 \%$ foetal bovine serum (Biowest, Nuaillé, France) and 100-units/mL penicillin and $100 \mu \mathrm{g} / \mathrm{mL}$ streptomycin (Sigma). All flasks were kept incubated at $37^{\circ} \mathrm{C}$ and in $5 \%$ CO2. MIA $\mathrm{PaCa}-2$ cells were discarded after being sub-cultured 30 times whereas HPSCs were discarded after 10.

\section{Drug preparation}

Stock solutions of specific PFKFB3 inhibitor 1-(4-Pyridinyl)-3-(2-quinolinyl)-2-propen-1-one (PFK15) were made up to $30 \mathrm{mM}$ in dimethyl sulfoxide (DMSO, both Sigma). PFK15 was further diluted in DMEM for experimentation. For calcium imaging experiments, PFK15 stock solutions were diluted directly in HEPES-buffered physiological saline solution (HEPES-PSS: $138 \mathrm{mM} \mathrm{NaCl}, 4.7 \mathrm{mM} \mathrm{KCl}, 10 \mathrm{mM}$ HEPES, $5.5 \mathrm{mM}$ glucose, $1.28 \mathrm{mM} \mathrm{CaCl} 2,0.56 \mathrm{mM} \mathrm{MgCl} 2$, pH 7.4). Calcium-free HEPES-PSS (0Ca-HEPES-PSS: 138 $\mathrm{mM} \mathrm{NaCl}, 4.7 \mathrm{mM}$ KCl,10 mM HEPES, $5.5 \mathrm{mM}$ glucose, $1.28 \mathrm{mM} \mathrm{CaCl} 2,0.56 \mathrm{mM} \mathrm{MgCl} 2,1 \mathrm{mM}$ EGTA pH 7.4) and high calcium HEPES-PSS $(138 \mathrm{mM} \mathrm{NaCl}, 4.7 \mathrm{mM} \mathrm{KCl}$, $10 \mathrm{mM}$ HEPES, $5.5 \mathrm{mM}$ glucose, $20 \mathrm{mM} \mathrm{CaCl} 2,0.56 \mathrm{mM}$ $\mathrm{MgCl} 2, \mathrm{pH}$ 7.4) were used for clearance assays. All drugs used in the metabolic inhibitor cocktail: iodoacetate $2 \mathrm{mM}$, (IAA), bromopyruvate $200 \mu \mathrm{M}$ (BrPy), oligomycin $10 \mu \mathrm{M}$ (OM) and carbonyl cyanide 3-chlorophenylhydrazone $4 \mu \mathrm{M}$ (CCCP) were obtained from Sigma-Aldrich and stock solutions dissolved in milliQ water (IAA) or DMSO. 


\section{Cell proliferation and viability assays}

Viable cells were counted using trypan blue staining and a haemocytometer. 2500 cells (5000 BxPC-3 cells) were seeded onto clear 96-well plates (Corning). Wells containing only media and vehicle acted as blanks. Plates were then incubated for $2-96 \mathrm{~h}$. At each time-point, $10 \mu \mathrm{L}$ of CCK-8 (Dojindo, Munich, Germany) was added to each well and plates were incubated for another $60 \mathrm{~min}$ at $37^{\circ} \mathrm{C}$. Following incubation, the plate was read for absorbance at $450 \mathrm{~nm}$ using a Synergy HT plate reader, operated by Gen 5 software (both Biotek, Whiting, Vermont, USA). Absorbance from blank wells containing only DMEM and vehicle were subtracted from each well reading. Cells were then fixed using of $100 \mu \mathrm{L} 10 \%$ trichloroacetic acid at $4{ }^{\circ} \mathrm{C}$ for $1 \mathrm{~h}$, washed with milliQ water and dried at $50^{\circ} \mathrm{C}$. Fixed cells were stained using $100 \mu \mathrm{L} 0.057 \%$ sulforhodamine-B (SRB) (Sigma) dissolved in 1\% acetic acid. Unbound dye was washed off of the cells using $1 \%$ acetic acid and plates were then dried at $50{ }^{\circ} \mathrm{C}$. Bound dye was re-suspended in 200- $\mu \mathrm{L}$ 10-mM Tris-Base solution (Sigma) and absorbance at $540 \mathrm{~nm}$ was read using the Synergy HT plate reader. There were 4 replicates for each condition in every experiment.

\section{Global ATP measurement}

MIA PaCa-2 cells were seeded at 10,000 cells/well into clear-bottomed, white-walled 96-well plates (Corning) and allowed to adhere overnight. The following day, wells were treated with PFK15, inhibitor cocktail $(4 \mu \mathrm{M}$ CCCP, $10 \mu \mathrm{M}$ oligomycin, $200 \mu \mathrm{M}$ bromopyruvate and 2 $\mathrm{mM}$ iodoacetate) or vehicle (DMSO). Global ATP was quantified using a Vialight plus assay (Lonza, Slough, UK), following the manufacturer's instructions, using a Biotek HT plate reader.

\section{Seahorse XFe analysis}

Both extracellular acidification rate (ECAR) and oxygen consumption rate (OCR) were measured simultaneously using a Seahorse XFe96 analyser (Agilent, CA, USA). MIA PaCa-2 cells, cultured as described above, were seeded in the manufacturer's 96-well plate at 20,000 cells/well and allowed to attach overnight. One hour before experimentation, media was changed to Seahorse XF DMEM medium pH 7.4 (Agilent), supplemented to contain $25 \mathrm{mM}$ glucose, $4 \mathrm{mM} \mathrm{L-}$ glutamine and $2 \mathrm{mM}$ pyruvate. The plate was allowed to equilibrate for $1 \mathrm{~h}$ in a $\mathrm{CO}_{2}$-free incubator at $37^{\circ} \mathrm{C}$ before loading into the Seahorse analyser. Seahorse XF96 cartridges were hydrated as per the manufacturer's instructions. Cells were pre-treated with either PFK15 or vehicle $20 \mathrm{~min}$ before the plate was loaded onto the analyser.

\section{Immunoblotting}

Cells were seeded onto 6-well plates (Corning) and allowed to adhere overnight. After treatment, cells were lysed with ice-cold lysis buffer (50 mM Tris-base, $40 \mathrm{mM}$ sodium pyrophosphate, $100 \mu \mathrm{M}$ sodium fluoride, $150 \mathrm{mM}$ sodium chloride, $1 \%$ Triton X-100, $10 \mathrm{mM}$ EGTA and EDTA sodium salt, $1 \mathrm{x}$ complete EDTA-free protease inhibitor, $1 \times$ phostop (Roche, Hertfordshire, UK). Lysates were subjected to sonication at an amplitude of $15 \mu \mathrm{m}$ using a soniprep-150 sonicator (Sanyo, Watford, UK). Protein was quantified using a BCA assay kit and Nanodrop 2000 (both Thermo Fisher, Waltham, Massachusetts, USA).

Anti-PFKFB3, GAPDH and anti-PARP monoclonal rabbit antibodies were used at a concentration of 1:1000 for probing (\#13123, \#5174 and \#9542S respectively Cell Signaling Technology). Mouse anti-PMCA4 (JA9, \# MA1-914, Thermofisher) was also used 1:1000. HRP conjugated goat anti-rabbit antibody (\#7074. Cell Signalling Technology) or HRP conjugated horse anti-mouse (\#7076 Cell Signalling Technology) were used as a secondary antibody. Clarity ECL, a Chemidock and ImageLab acquisition software were used to produce digital images (BioRad). Image J software was used to quantify an PARP:cleavedPARP band volumes in MIA PaCa-2, BxPC-3 and HPSCs after $6 \mathrm{~h}$ of treatment with $10 \mu \mathrm{M}$ PFK15.

\section{Membrane protein isolation}

Membrane and cytosolic proteins were separated using a plasma membrane protein extraction kit (Abcam, \#65400). Approximately $2 \times 10^{8}$ cells were grown in 160 $\mathrm{mm}$ plates and were treated as per the manufacturer's instructions. Both membrane and cytosolic fractions were then assessed by Immunoblot.

\section{Calcium imaging}

MIA PaCa-2 cells and HPSCs were seeded onto $16-\mathrm{mm}$ or 25-mm glass coverslips in non-treated 6-well plates (Corning) containing $25 \mathrm{mM}$ glucose DMEM. These cells were then incubated for $24 \mathrm{~h}$ to allow adherence to the coverslip. Cells were then loaded with $5 \mu \mathrm{M}$ Fura-2AM (Teflabs, Austin, Texas, USA) as previously described [9]. Two microscopes were used for imaging, a Nikon Diaphot and a Nikon TE2000S and images were obtained using an Orca CCD (Hamamatsu, Hamamatsu, Shizuoka, Japan) CoolSNAP HQ progressive-scan CCD camera (Roper Scientific, Sarasota, Florida, USA) respectively. Excitation was performed using a monochromator illumination system (Cairn Research, Faversham, UK). All image acquisition was controlled by Metafluor software (Molecular Devices, San Jose, California, USA), including both excitation and data recording. Cells were perfused with either standard HEPES- 
PSS (138 mM NaCl, $4.7 \mathrm{mM} \mathrm{KCl,} 1.28 \mathrm{mM} \mathrm{CaCl} 2,0.56$ $\mathrm{mM} \mathrm{MgCl}$, $5.5 \mathrm{mM}$ glucose, $10 \mathrm{mM}$ HEPES, $\mathrm{pH} 7.4$ ), or HEPES-PSS containing a drug, using a gravity-fed perfusion apparatus (Harvard Apparatus, Holliston, Massachusetts). Calcium overload and clearance experiments were conducted as previously described as was calcium calibration $[9,10,36]$.

\section{Data analysis}

All data analysis was conducted using Prism 7 software (GraphPad) and Microsoft Excel (Microsoft). Data was tested for normal distribution using a KolmogorovSmirnoff test and the outcome determined whether a parametric or non-parametric statistical test was used and a $p$ value below 0.05 was considered significant.

\section{Results}

\section{PFKFB3 is overexpressed in PDAC and predicts poor} prognosis

Expression of PFKFB1-4 and PFKP in tumour tissue versus healthy pancreatic epithelia was assessed by interrogating the Oncomine database (www.oncomine.com, Thermo Fisher Scientific, Ann Arbor, MI) and array dataset: Badea Pancreas [35]. This demonstrated that PFKFB3 and PFKP were overexpressed in tumour tissue, whilst there was modest upregulation of PFKFB2 and expression of PFKFB1 and PFKFB4 was downregulated, compared with healthy tissue from the same pancreatic resection $(n=39, p<$ 0.0001, Fig. 1a/b). Next, the relationship between PFKFB3 expression and overall survival was assessed using expression data from 51 PDAC patients using the PROGgeneV2 software [37, 38], Indiana University Purdue University, Indianapolis, IN) and survival data from PFKFB3 expression was bifurcated at the median and survival was assessed using the Kaplan-Meier method and PROGgeneV2's inbuilt proportional hazards analysis. Patients with low PFKFB3 expression lived significantly longer than patients with high PFKFB3 expression (hazard ratio $=1.64$, $p=0.0033$; Fig. 1c).

PFK15 reduces cell proliferation and induces cell death in PDAC MIA PaCa-2 cells

Glycolytic ATP is required for PMCA function; inhibition of glycolysis using iodoacetate (IAA) and bromopyruvate (BrPy) causes cytotoxic calcium overload and cell death in PDAC cells $[9,10]$. To assess whether PFKFB3, a known driver of the Warburg phenotype, is required for this phenomenon, the specific PFKFB3 inhibitor PFK15 was

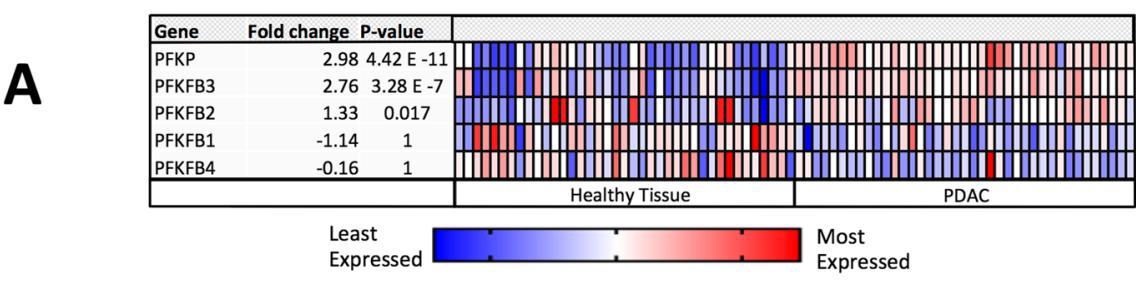

B

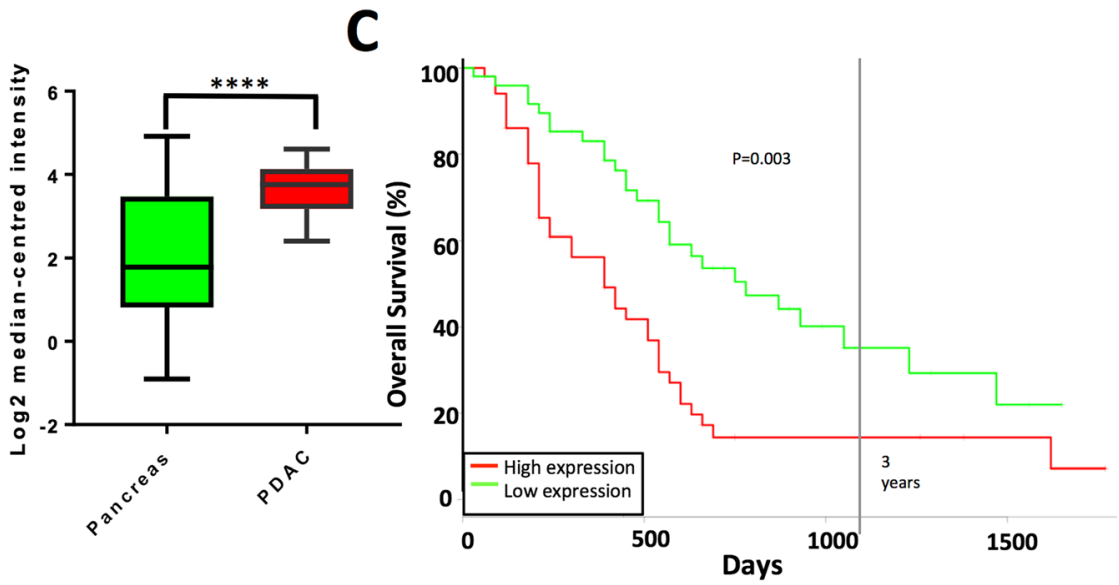

Fig. 1 Expression of PFKFB3 is upregulated and correlates with poor prognosis in PDAC: a Heat map analysis of overexpression of PFKFB isoforms 1-4 and PFKP in tumour tissue versus healthy pancreatic tissue in the pancreas resected from PDAC patients (datamined from Badea Pancreas dataset using Oncomine [35]) b Box and whisker plot displaying PFKFB3 expression in tumour tissue versus healthy tissue from resected tissue (unpaired $t$ test; $n=39$ ). c Survival rates of PDAC patients with high or low expression of PFKFB3 estimated using the Kaplan-Meier method and proportional hazards analysis; $n=51, p=0.003$ (figure generated using PROGgeneV2, *** $p<0.0005$ ) 


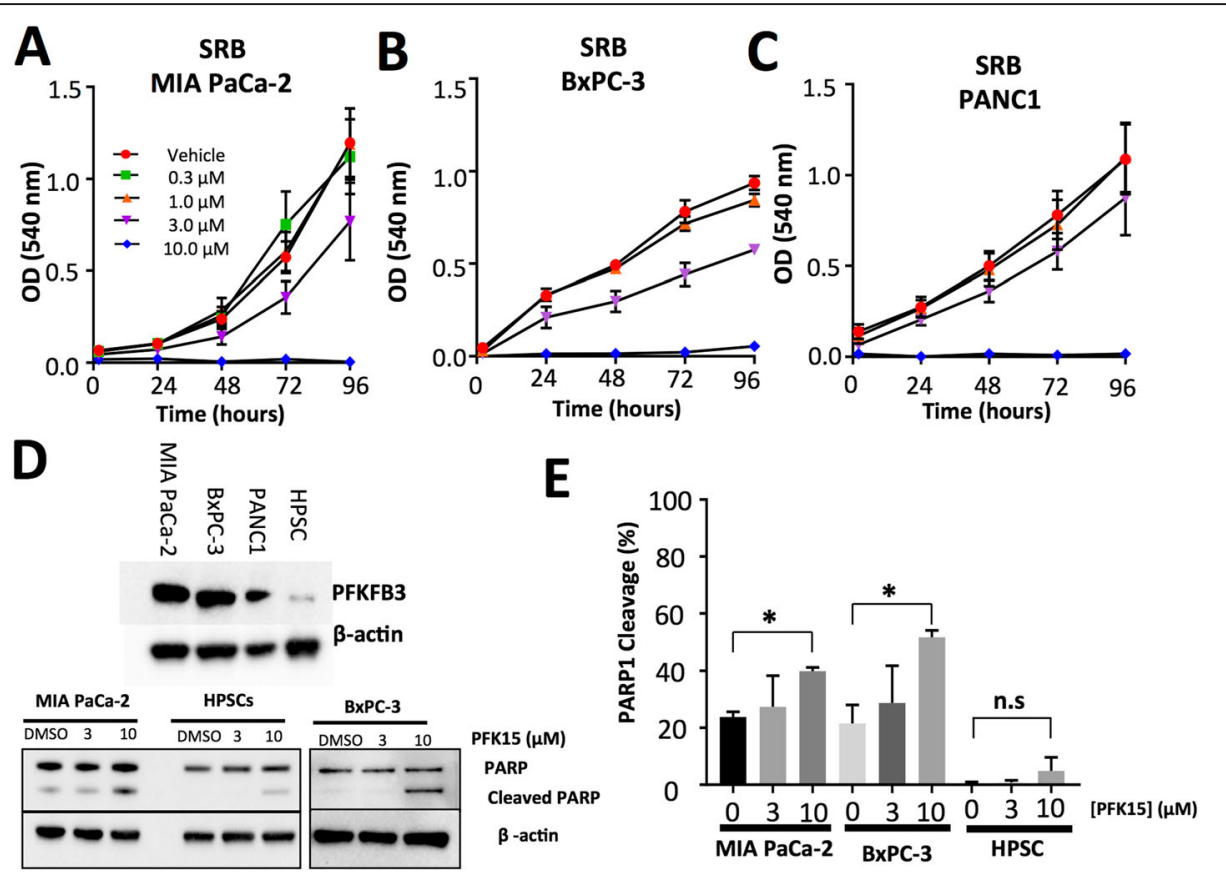

Fig. 2 PFK15 reduces proliferation and induces death in MIA PaCa-2 but not human pancreatic stellate cells: PDAC cell lines:MIA PaCa-2, BxPC-3 and PANC1 were treated with 0.3-10 $\mu \mathrm{M}$ PFK15 for up to $96 \mathrm{~h}$; cell proliferation was measured using a sulforhodamine-B assay (a-c). Western blot for PFKFB3 in MIA PaCa-2, BxPC-3 and human pancreatic stellate cells (HPSCs) (di). Western blot for PARP cleavage (dii), cells were treated with PFK15 (3-10 $\mu \mathrm{M})$ or vehicle (DMSO) for $6 \mathrm{~h}$ and bands were quantified; the intensity of cleaved PARP is expressed as a percentage of total PARP (e). Points and bars represent the mean \pm SEM of 5 separate experiments. Kruskall-Wallis test; $p<0.05^{*}$

used. The effects of PFK15 on cell proliferation and cell viability were assessed in PDAC cell lines MIA PaCa-2, BxPC3 and PANC1 using a sulforhodamine-B (SRB) assay (Fig. 2ac) and tetrazolium-based cell counting kit (CCK8, Additional file 1: Figure S1A-C). In SRB assays, $10 \mu \mathrm{M}$ PFK15 produced a significant reduction in MIA PaCa-2 cell proliferation after $72 \mathrm{~h}$ (Fig. 2a). However, the SRB assay is limited by numerous wash steps that can lead to loss of poorly adhered cells, and thus underestimation of cell number. Therefore, any drug that affected cell adhesion, independent of proliferation, would compromise the validity of conclusions drawn. To address this, we used an accompanying CCK8 assay, which measures the redox potential of live cells, independent of cell adherence. Results from CCK8 assays closely mirrored SRB results indicating that changes observed were real (Fig. 2b).

In both of these assays, it is difficult to distinguish between reduced proliferation and cell death and, based on the steep concentration-effect relationship observed, it is possible that $10 \mu \mathrm{M}$ PFK15 induces cell death. Therefore, apoptosis was assessed by immunoblot for poly (ADP-ribose) polymerase (PARP), a cleavage target of caspase 3 during apoptosis $[39,40]$. PARP cleavage is a marker of apoptosis and thus the ratio of cleaved: whole PARP was assessed in MIA PaCa-2, BxPC-3 and human pancreatic stellate cells (HPSCs), as a non-cancer control with much lower PFKFB3 expression (Fig. 2di). Treatment with $10 \mu \mathrm{M}$
PFK15 for $24 \mathrm{~h}$ caused a significant increase in PARP cleavage in MIA PaCa-2 and BxPC-3 when compared with vehicle-treated cells (Kruskall-Wallis test: MIA PaCa-2 vehicle: $25.07 \pm 1.91,10 \mu \mathrm{M}$ PFK15: $40.46 \pm 1.19 p=0.032$, $n=5$, BxPC3 vehicle: $21.51 \pm 6.57,10 \mu \mathrm{M}$ PFK15: $51.80 \pm$ 2.44, $n=3 p=0.016$ Fig. 2dii/e). MIA PaCa-2 cells were selected going forward, these cells have high PFKFB3 expression, high growth rate and have been identified as highly glycolytic in comparison to other PDAC cell lines; they also express mutant p53 and mutant K-RAS, the most common mutations in PDAC $[9,10,41-43]$.

\section{PFK15 produces calcium overload in MIA PaCa-2 but not human pancreatic stellate cells}

Previously, glycolytic inhibitors have been shown to produce cytotoxic calcium overload in PDAC cell lines [9]. We sought to assess whether this was the case for specific PFKFB3 inhibitor PFK15. Fura-2 loaded MIA PaCa-2 cells were perfused with HEPES-buffered physiological saline solution (HEPES-PSS) with or without $10 \mu \mathrm{M}$ PFK15 for 40 min, during which $\left[\mathrm{Ca}^{2+}\right]_{\mathrm{i}}$ was measured using fura-2 imaging. As expected, untreated MIAPaCa-2 cells were able to maintain $\left[\mathrm{Ca}^{2+}\right]_{\mathrm{i}}$ at a relatively constant resting level for the duration of the experiment, however $\left[\mathrm{Ca}^{2+}\right]_{\mathrm{i}}$, increased significantly after PFK15 treatment $(p<0.0001$; Fig. 3). To determine whether this effect was due to PFKFB3 inhibition, HPSCs, which have very low PFKFB3 

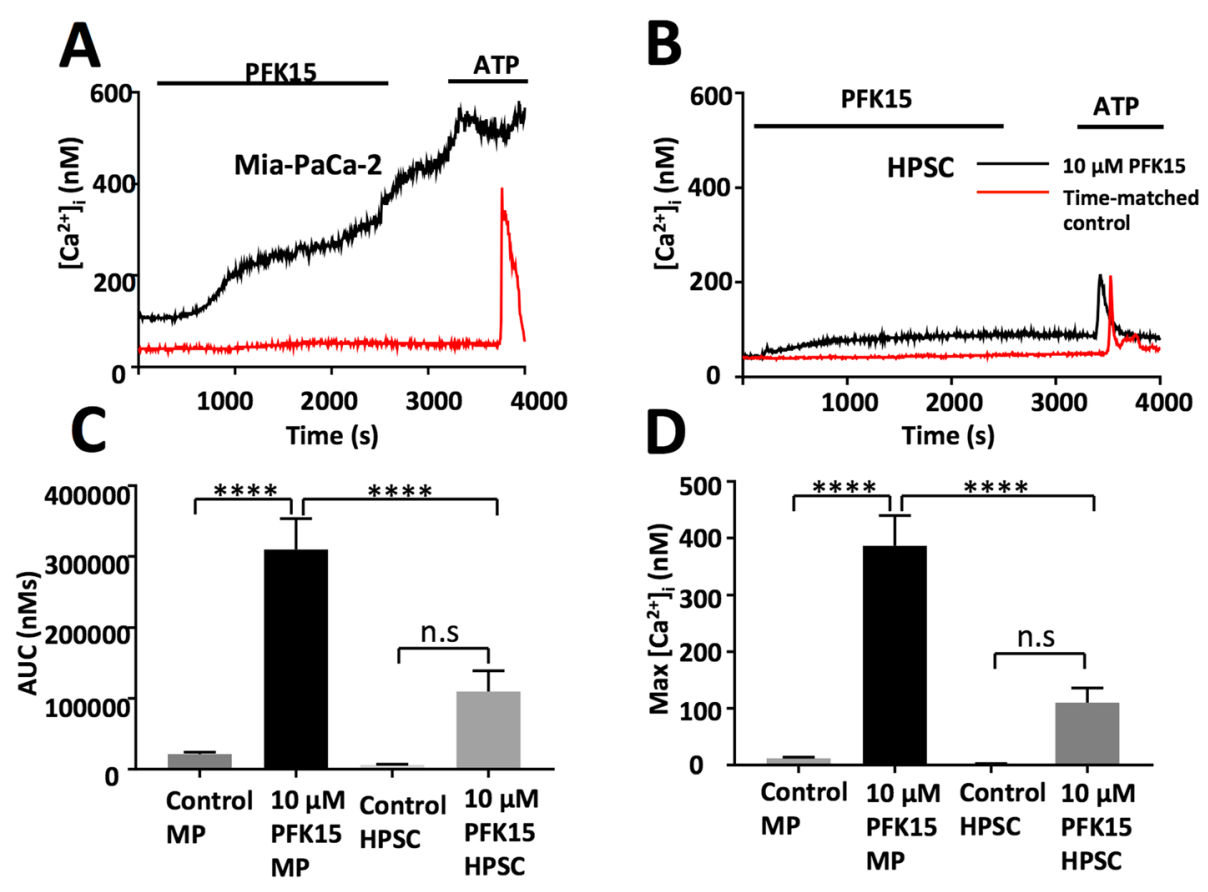

Fig. 3 PFK15 causes calcium overload in MIA PaCa-2 but not HPSC: Fura-2 fluorescence microscopy was used to assess the effects of PFK15 on intracellular calcium $\left(\left[\mathrm{Ca}^{2+}\right]_{\mathrm{i}}\right)$ in MIA PaCa-2 cells (a) and human pancreatic stellate cells (HPSCs) (b). Cells were loaded with fura-2 dye and perfused with HEPES-PSS or HEPES-PSS containing $10 \mu \mathrm{M}$ PFK15 for 40 min then HEPES-PSS containing $100 \mu \mathrm{M}$ ATP. Traces were assessed for area under the curve (c) and maximum calcium increase (d). a and $\mathbf{b}$ are representative traces for MIA PaCa-2 and HPSCs respectively, $\mathbf{c}$ and $\mathbf{d}$ represent the mean \pm SEM, $n=3-12$ separate experiments, one-way ANOVA, ${ }^{* * *} p<0.0005$

expression, were assessed in the same way and no significant calcium overload was observed (Fig. 3).

\section{PFK15 inhibits PMCA activity in MIA PaCa-2 but not human pancreatic stellate cells}

Previously, the effect of glycolytic inhibition on PMCA function has been assessed following functional and pharmacological isolation of PMCA activity [9, 10]. Cells were subjected to ER calcium depletion using SERCA inhibitor cyclopiazonic acid (CPA, $30 \mu \mathrm{M})$ throughout the experiment, in calcium-free HEPES-PSS containing $1 \mathrm{mM}$ EGTA (0Ca-HEPES-PSS). Store-operated calcium entry occurs when perfusion is switched to $20 \mathrm{mM}$ calcium HEPES-PSS; when calcium is removed and the clearance is mediated by PMCAs [9, 10, 44, 45]. This is repeated twice per experiment and the clearance rate of the fastest 2-min portion of the first clearance phase $\left(R_{1}\right)$ is compared with the second clearance rate from the same fura- 2 ratio $\left(R_{2}\right)$, clearance is assumed to be linear during this phase; however, any trace that produced an $\mathrm{R}$-squared value below 0.9 was excluded. This method was employed to test the effects of PFK15 and distinguish whether PMCA inhibition was responsible for PFK15-induced calcium overload. Both MIA PaCa-2 and HPSCs were able to conserve PMCA-mediated clearance with 30 min of 0Ca-HEPES-PSS between clearance phases, in time-matched controls; producing mean $R_{2} / R_{1}$ values of $0.98 \pm 0.02(n=5)$ and $0.87 \pm 0.11(n=3)$ respectively. When $10 \mu \mathrm{M}$ PFK15 was added for $30 \mathrm{~min}$ between clearance phases, a significant reduction of clearance rate was observed in MIA PaCa-2 but not HPSCs when compared with time-matched controls $\left(\mathrm{R}_{2} / \mathrm{R}_{1}=0.52 \pm 0.04 \quad(n=5) \quad\right.$ and $0.76 \pm 0.06 \quad(n=4)$ respectively, Fig. $4 \mathrm{a}-\mathrm{c})$.

PFKFB3 is localised to the plasma membrane in PDAC In order to determine whether a pool of PFKFB3 is localised to the plasma membrane, in close proximity to PMCAs, a plasma membrane protein isolation kit was employed and samples were assessed by immunoblot. This kit is designed to specifically isolate plasma membrane proteins using a series of spin steps and produces lysates with over 90\% purity. Membrane samples from MIA PaCA-2 cells and HPSCs were verified using PMCA4 as a positive control whilst GAPDH was used as a cytosolic control (Fig. 4d). As expected, PFKFB3 was again shown to be much lower in HPSCs compared with MIA PaCa-2 cells. PFKFB3 was identified in the plasma membrane fraction of MIA PaCa-2 but not HPSCs. Even when the membrane was overexposed, PFKFB3 was not detected in the membrane of HPSCs (Fig. 4d). 


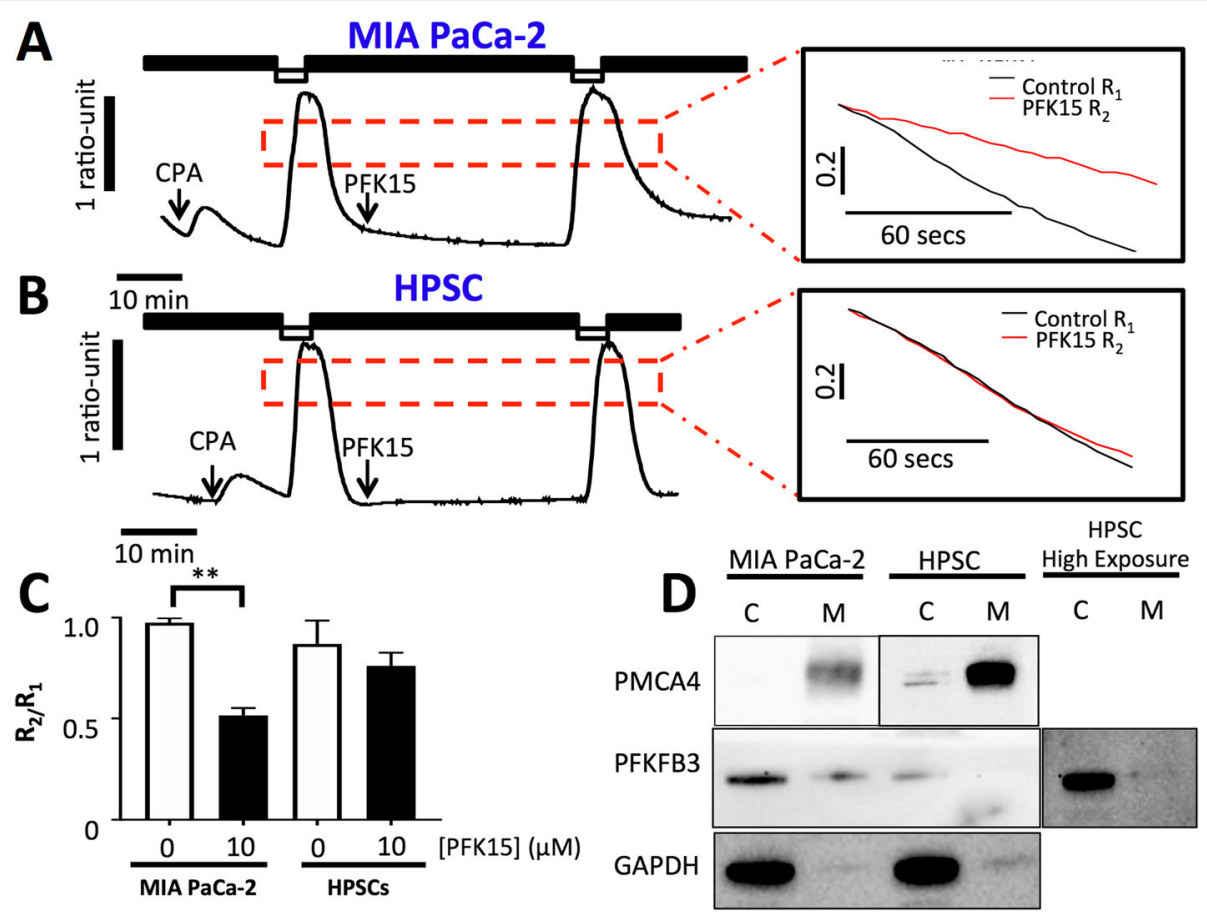

Fig. 4 PFK15 causes PMCA inhibition in MIA PaCa-2 but not human pancreatic stellate cells: PMCA activity was measured in MIA PaCa-2 cells and HPSCs perfused under constant SERCA blockade (cyclopiazonic acid, CPA, $30 \mu \mathrm{M}$ ) and calcium-free conditions and $1 \mathrm{mM}$ EGTA (black bar). Subsequent ER Ca ${ }^{2+}$ depletion caused store-operated calcium entry when $20 \mathrm{mM} \mathrm{Ca}^{2+}$ was introduced (white bar) calcium efflux was used as a measure of PMCA activity before and after 30 min incubation with or without $10 \mu M$ PFK15. Representative traces showing clearance experiments with PFK15 in MIA $\mathrm{PaCa}-2(\mathbf{a})$ and HPSCs (b). The rate of clearance was calculated for the fastest 2 min of the first clearance phase $\left(R_{1}\right)$, the clearance rate from the same starting ratio was then calculated in the second clearance phase $\left(R_{2}\right) . R_{1} / R_{2}$ was calculated for both MIA PaCa-2 and HPSC with and without PFK15 treatment (c). Membrane and cytosolic protein samples from MIA PaCa-2 cells were produced using a membrane protein extraction kit and PFKFB3 levels assessed by immunoblot. PMCA4 was used as a positive control for membrane samples and GAPDH was used as a positive control for cytoplasmic samples, a representative image is shown (d). Bars represent the mean \pm SEM of 3-5 experiments. Kruskall-Wallis test; ${ }^{* *} p<0.005$

PFK15 reduces glycolysis and mitochondrial respiration but does not affect global ATP levels in PDAC

In order to assess whether the effects of PFK15 on cell proliferation and $\left[\mathrm{Ca}^{2+}\right]_{\mathrm{i}}$ in MIA PaCa-2 cells were due to metabolic inhibition, the effects on glycolysis (extracellular acidification, ECAR) and mitochondrial metabolism (oxygen consumption, OCR) were assessed using a Seahorse XFe analyser. Due to solubility issues with injection, MIA PaCa-2 cells were pre-treated with $10 \mu \mathrm{M}$ PFK15 for 20 min and then ECAR and OCR were measured, simultaneously. Cells treated with PFK15 showed significantly lower steady-state ECAR and OCAR at all time points compared to control (unpaired $t$ test $p<0.05, n=3$ ). Both ECAR and OCR remained stable in control cells for the duration of the experiment (Fig. 5a/b).

Next, we wanted to test whether this change in ECAR/ OCR translated to ATP depletion. This was assessed using a luciferase-based assay kit. MIA PaCa-2 cells were treated with PFK15 $(3 \mu \mathrm{M}, 10 \mu \mathrm{M})$, ATP depletion cocktail $(4 \mu \mathrm{M}$ CCCP, $10 \mu \mathrm{M}$ oligomycin, $200 \mu \mathrm{M}$ bromopyruvate and 2 $\mathrm{mM}$ iodoacetate) or vehicle (DMSO) for 1-6h. Based on the results of calcium overload experiments, it was hypothesised that $1 \mathrm{~h}$ would allow enough time for the drug to exert any effects on ATP that subsequently cause a cytotoxic calcium overload. Surprisingly, PFK15 caused no significant global ATP depletion even after 1 or $5 \mathrm{~h}$ of PFK15 treatment, whereas the ATP depletion cocktail caused close to $100 \%$ depletion in all experiments (Fig. $5, n=4$ ).

\section{Discussion}

The present study is the first to demonstrate a link between PFKFB3 and calcium homeostasis in PDAC and supports the hypothesis that targeting glycolysis is a viable treatment in PDAC. Glycolytic driver PFKFB3, which has the highest kinase activity of the PFKFB family, is overexpressed in PDAC, whereas PFKFB isoforms which oppose aerobic glycolysis are downregulated (Fig. 1). We have identified that the PFKFB3 inhibitor, PFK15, causes cytotoxic calcium overload via inhibition of PMCA function, ultimately leading to cell death. This effect was not observed in non-cancerous HPSCs. This is of therapeutic importance as any new therapeutic strategy for PDAC must be specific. PFK15 has previously been effective in reducing tumour growth when 


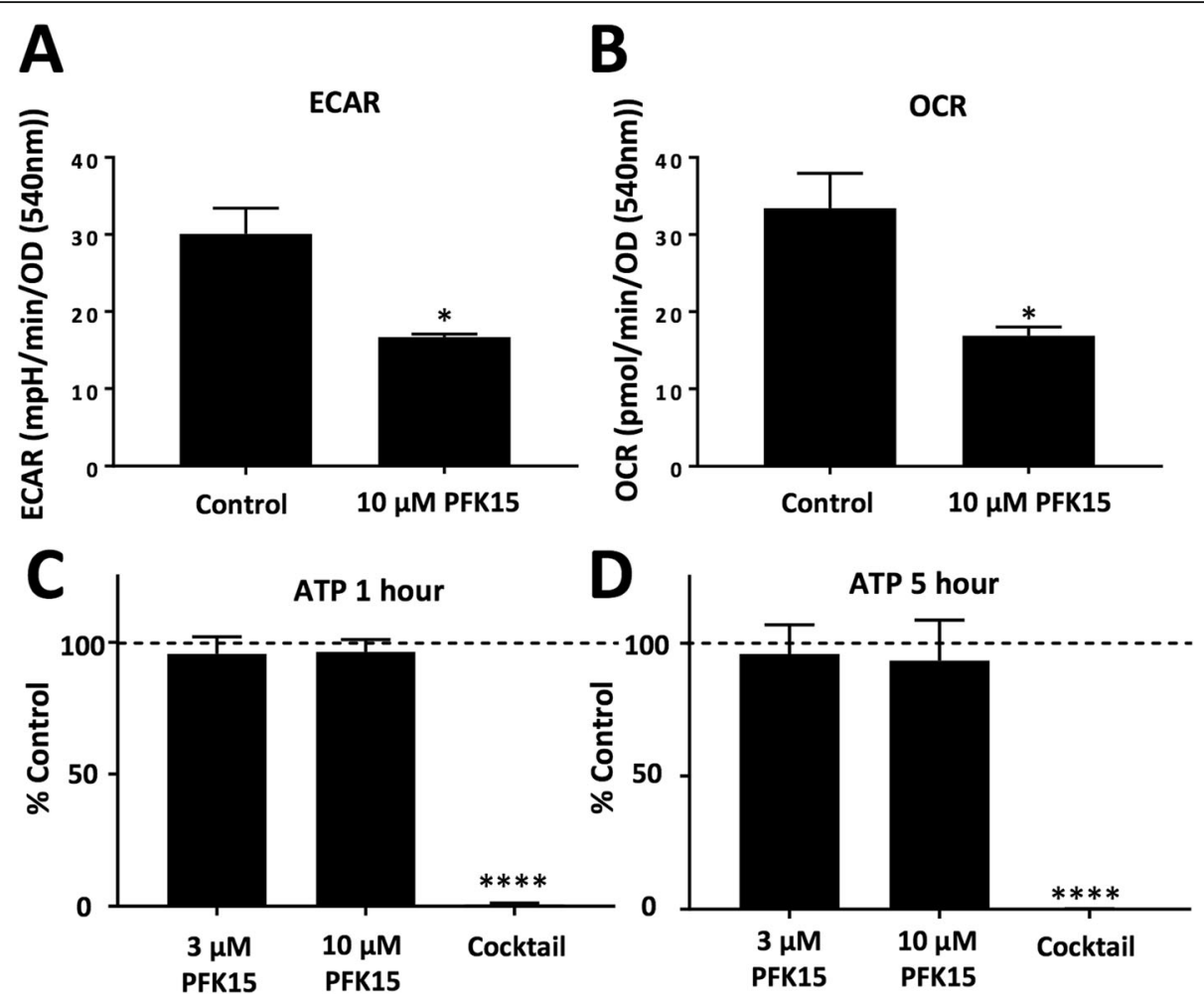

Fig. 5 PFK15 reduces both ECAR and OCR but does not cause ATP depletion in MIA PaCa-2 cells: a, b: MIA PaCa-2 cells were treated with $10 \mu \mathrm{M}$ PFK15 or vehicle (DMSO) for 20 min before loading onto a seahorse XFe analyser. Extracellular acidification rate (ECAR, a) and oxygen consumption rate $(\mathrm{OCR}, \mathbf{b})$ were recorded after 5 min. MIA PaCa-2 cells were treated for 1 (c) or 5 (d) h with PFKFB3 inhibitor PFK15 (3 or $10 \mu \mathrm{M})$, or a cocktail containing $4 \mu \mathrm{M} \mathrm{CCCP}, 10 \mu \mathrm{M}$ oligomycin, $200 \mu \mathrm{M}$ bromopyruvate and $2 \mathrm{mM}$ iodoacetate as a positive control. ATP was measured using a luciferase-based assay kit and replicates were averaged and normalised to vehicle-treated, time-matched control. Data presented are the mean \pm SEM, $n=4$ separate experiments, each containing 4-8 replicates. Unpaired $t$ test/ Kruskall-Wallis test; ${ }^{*} p<0.05$

administered to athymic mice with PDAC (BxPC-3), glioblastoma (U-87 MG) and colon adenocarcinoma (CT26) xenografted tumours. Importantly, PFK15 did not have severe side effects, despite PFKFB3 expression in the muscle, adipose tissue and other tissues [24, 46]. The present study provides further evidence to support the hypothesis that PFK15 has the potential to be used as an anti-cancer drug in PDAC.

Previous work leading to this study demonstrated that glycolytic inhibitors, but not mitochondrial inhibitors cause significant global ATP depletion and the consequent inhibition of PMCA activity and calcium overload $[9,10]$. In the present study, PFK15 induced inhibition of the PMCA, calcium overload and cell death in PDAC cells, without any effect on steady-state global ATP. Moreover, in Seahorse XF experiments PFK15 reduced both ECAR, which is expected for a bona fide glycolytic enzyme inhibitor, but also reduced OCR, which was more unexpected as one would expect that a glycolytic inhibitor would cause a compensatory increase in OCR in an attempt to preserve cellular ATP. Although we cannot rule out that PFK15 may have previously unreported non-specific effects on mitochondrial function, it is important to emphasise that our previous studies have shown that mitochondrial inhibitors, which cause profound inhibition of OCR, do not affect cell survival/ growth, reduce ATP or inhibit PMCA activity $[9,10]$. This, therefore, suggests that the effects of PFK15 on PMCA activity are due to inhibition of glycolysis, rather than mitochondrial function. Nevertheless, the effect of PFK15 on ECAR and OCR without affecting steady-state ATP presents a functional paradox which is difficult to reconcile. However, a possible explanation is that the steady-state ATP concentration within the cell is dependent not only on the ATP production rate but also on the ATP consumption rate. Given that PFK15 inhibits cell proliferation and is reported to arrest the cell cycle [26, 30]; which are major ATP consuming processes, it is possible that reduced ATP production, due to inhibition of glycolysis, coincides with a corresponding reduced ATP consumption; such that steady-state ATP is maintained. Furthermore, in the context of inhibition of the PMCA, it is also possible that PFK15 inhibits a significant pool of PFKFB that resides in close proximity to the PMCA at the membrane, where it provides a privileged ATP supply to 
the PMCA without necessarily affecting global steadystate ATP.

Indeed, in the present study, PFKFB3 was identified in the plasma membrane fraction, indicating localisation of a significant pool of PFKFB3 at the plasma membrane. This further supports the above hypothesis that PFK15 inhibits this pool of PFKFB3, thereby reducing glycolytic flux and thus localised supply of ATP to the PMCA. ATP is lower in cancer cells than in healthy tissues due to the Warburg effect and high expression of PKM2. PKM2 is overexpressed in PDAC but has low catalytic activity. This causes a bottleneck in glycolysis that allows the accumulation of glycolytic intermediates, which are utilised for anabolic processes, at the expense of ATP production [12, 14-17]. It, therefore, makes teleological sense that glycolytic enzymes, as the major source of ATP in cancer cells, are localised to where ATP is required; in close proximity to ion pumps at the plasma membrane. Ion pumps such as PMCAs and the $\mathrm{Na}^{+} / \mathrm{K}^{+}$ATPase are major ATP consumers in all cells, including cancer cells. This localisation would also facilitate glycolytic flux as ATP would be consumed by ion pumps soon after production. This would keep ATP concentration below the inhibitory threshold of PFK1, thereby maintaining glycolytic flux. Such localisation has been previously described in erythrocytes, which have no mitochondria and therefore exclusively require glycolytic ATP to fuel PMCAs [47]. Experiments using plasma membrane vesicles from pig smooth muscle have found that PMCA function is maintained when glycolytic substrates are provided, indicating that glycolytic enzymes can associate with the membrane in mammalian cells [48]. Moreover, the platelet isoform of PFK1 (PFKP) is recruited to the membrane by activated EGFRs and is overexpressed in PDAC (Fig. 1a) [22], whilst the liver isoform of PFK1 (PFKL) has been found to oligomerize to form filaments which were enriched at the plasma membrane [49].

Caveolae are caveolin-1 rich invaginations in the plasma membrane, in which proteins are held in close proximity allowing close-communication between signalling complexes and can also function to assist endocytosis and exocytosis [50, 51]. PMCAs are found in caveolae, which allows regulation of PMCA activity and may allow association of PMCAs with glycolytic enzymes [52-56]. Enolase, a glycolytic enzyme has been reported to localise to the plasma membrane and in particular, caveolae [57]. Therefore, caveolae may facilitate the assembly of a glycolytic 'metabolon' (complex of enzymes) at the membrane and in close proximity to PMCAs. Membrane association of glycolytic enzymes could explain why glycolytic but not mitochondrial inhibitors affect PMCA function. The ATP demands of membrane-bound pumps such as PMCAs and $\mathrm{Na}^{+} / \mathrm{K}^{+}$ATPases are so high that the source of ATP must be in close proximity in order to maintain function.
Increasing the ATP demand of $\mathrm{Na}^{+} / \mathrm{K}^{+}$ATPases causes an increase in glycolysis but not OXPHOS, whilst inhibition of $\mathrm{Na}^{+} / \mathrm{K}^{+}$ATPases has the opposite effect on glycolysis [58]. Castro et al. have also demonstrated that membrane pumps have a local ATP supply in HeLa cells and that, when ATP is low, it is preferentially used by $\mathrm{Na}+/ \mathrm{K}+$ ATPase pumps, leading to impaired PMCA activity. Inhibition of $\mathrm{Na}+/ \mathrm{K}+$ ATPase pumps using ouabain rescues this effect. The authors described this as 'ATP steal'. In relation to the present study, this shows that PMCAs can be inhibited by sub-maximal ATP depletion and may be highly sensitive to localised ATP depletion [59]. PMCAs also have a low-affinity ATP binding site, where ATP binding increases PMCA activity. In cell-free assays, the reported $K_{m}=330 \mu \mathrm{M}[60,61]$. This value is likely to be higher in vitro. Taken together, this suggests that cancer cells may be much more vulnerable to PMCA inhibition than healthy cells.

Glycolytic ATP fuelling of PMCAs presents a novel therapeutic target for the treatment of cancer. As PMCAs are ubiquitously expressed, they cannot be targeted directly as this would have adverse effects on healthy tissues. However, glycolysis provides a 'preferential' ATP supply to PMCAs in PDAC. This may be due to association or co-localisation of glycolytic enzymes with PMCAs and/or the plasma membrane, providing a privileged ATP supply to fuel PMCAs, maintaining PMCA activity which is critical for cell survival.

In summary, PFKFB3 is overexpressed in PDAC where a pool is located at the plasma membrane. Inhibition of PFKFB3 in the PDAC cell line MIA PaCa-2 causes inhibition of PMCA function, which leads to cytotoxic calcium overload and cell death. These effects are independent of global ATP. Importantly, these effects are not observed in non-cancerous HPSCs suggesting that this phenomenon is cancer-specific. Taken together, this study describes a phenomenon that could represent a future therapeutic target for PDAC and other highly glycolytic cancers and also provides further evidence for PFK15 as an anti-cancer drug. Further understanding of this phenomenon may bring to light more novel therapeutic targets for the treatment of PDAC and other cancers.

\section{Conclusions}

PFKFB3 is overexpressed in PDAC where a pool is located at the plasma membrane. Inhibition of PFKFB3 in the PDAC cell line MIA PaCa-2 causes inhibition of PMCA function, which leads to cytotoxic calcium overload and cell death. These effects are independent of global ATP. Importantly, these effects are not observed in noncancerous HPSCs suggesting that this phenomenon is cancer-specific. Taken together, this study describes a phenomenon that could represent a future therapeutic 
target for PDAC and other highly glycolytic cancers and also provides further evidence for PFK15 as an anti-cancer drug. Further understanding of this phenomenon may bring to light more novel therapeutic targets for the treatment of PDAC and other cancers.

\section{Supplementary information}

Supplementary information accompanies this paper at https://doi.org/10. 1186/s40170-020-0210-2

Additional file 1: Figure S1. PFK15 reduces cell proliferation and induces cell death in PDAC cells but not human pancreatic stellate cells.

\section{Abbreviations}

$\left[\mathrm{Ca}^{2+}\right]$ : Intracellular calcium concentration; ATP: Adenosine triphosphate; BrPy: Bromopyruvate; CaM: Calmodulin; CCK-8: Cell counting kit 8 (dojindo); CPA: Cyclopiazonic acid; F16BP: Fructose-1,6-bisphosphate; F26BP: Fructose2,6-bisphosphate; F6P: Fructose-6-phosphate; GLUT1: Glucose transporter 1; HPSC: Human pancreatic stellate cell (cell line); IAA: lodoacetate;

OM: Oligomycin; OXPHOS: Oxidative phosphorylation; PARP: Poly-ADP-ribose polymerase; PDAC: Pancreatic ductal adenocarcinoma;

PFK: Phosphofructokinase; PFKFB: Phosphofructokinase-fructosebisphosphatase; PK: Pyruvate kinase; PMCA: Plasma membrane calcium ATPase; PSS: Physiological saline solution; SRB: Sulforhodamine-B

\section{Acknowledgements}

Authors would also like to thank Professor David Yule (University of Rochester, NY, USA) for providing HPSCS and Professor Mark Dunne for the use of his plate reader.

\section{Authors' contributions}

DAR conducted the majority of experiments and analysis for Figs. 1, 2, 3, 4 and 5 and prepared the first draft of the final manuscript. ADJ and AS conducted experiments for Figs. 3 and 4. PS provided vital assistance with Seahorse experiments and experimental design throughout. JIEB conceived and supervised the project, assisting in all experimental design and manuscript preparation. All authors read and approved the final manuscript.

\section{Funding}

This work was supported by a Pancreatic Cancer Research Fund (PCRF) grant and Medical Research Grant (MRC) grant (MR/P00251X/1) awarded to JIEB. DAR was funded by a University of Manchester Alumni Association Research Impact Fund and Doctoral Academy PhD scholarship. PS was funded by a Ministry of Science and Technology Royal Thai Government PhD scholarship and AS was funded by a Princess Nora University scholarship (Saudi Arabia) PhD scholarship.

\section{Availability of data and materials}

All analysed and derivative raw data available on request.

\section{Ethics approval and consent to participate}

There were no experiments performed on animals or human subjects or involving human tissue that required ethical consent.

\section{Consent for publication}

All authors consent to publish.

\section{Competing interests}

The authors declare that they have no competing interests.

\section{Author details}

'Division of Cancer Sciences, School of Medical Sciences, University Of Manchester, Michael Smith Building, Oxford Road, Manchester M13 9PT, UK. ${ }^{2}$ Department of Biology, University of York, Heslington, York, UK.
Received: 3 July 2019 Accepted: 12 January 2020

Published online: 02 April 2020

\section{References}

1. Siegel RL, Miller KD, Jemal A. Cancer statistics, 2018. CA Cancer J Clin. 2018; 68(1):7-30.

2. Siegel RL, Miller KD, Jemal A. Cancer statistics, 2015. CA Cancer J Clin. 2015; 65(1):5-29.

3. Li HY, Cui ZM, Chen J, Guo XZ, Li YY. Pancreatic cancer: diagnosis and treatments. Tumor Biol. 2015;36(3):1375-84.

4. Warburg O. On the metabolism of cancer cells. Naturwissenschaften. 1924; 12:1131-7.

5. Hanahan D, Weinberg RA. Hallmarks of Cancer: the next Generation. Cell. 2011;144(5):646-74.

6. Pavlova NN, Thompson CB. The emerging hallmarks of Cancer metabolism. Cell Metab. 2016;23(1):27-47.

7. San-Millan I, Brooks GA. Reexamining cancer metabolism: lactate production for carcinogenesis could be the purpose and explanation of the Warburg effect. Carcinogenesis. 2017;38(2):119-33.

8. Vander Heiden MG, Cantley LC, Thompson CB. Understanding the Warburg effect: the metabolic requirements of cell proliferation. Science. 2009; 324(5930):1029-33.

9. James AD, Chan A, Erice O, Siriwardena AK, Bruce JIE. Glycolytic ATP fuels the plasma membrane calcium pump critical for pancreatic Cancer cell survival. J Biol Chem. 2013;288(50):36007-19.

10. James AD, Patel W, Butt Z, Adiamah M, Dakhel R, Latif A, et al. The plasma membrane calcium pump in pancreatic Cancer cells exhibiting the Warburg effect relies on glycolytic ATP. J Biol Chem. 2015;290(41):24760-71.

11. Filipp FV. Cancer metabolism meets systems biology: Pyruvate kinase isoform PKM2 is a metabolic master regulator. J Carcinog. 2013;12:14.

12. Wong N, Ojo D, Yan J, Tang D. PKM2 contributes to cancer metabolism. Cancer Lett. 2015;356(2):184-91.

13. Li CG, Zhao ZM, Zhou ZP, Liu R. PKM2 promotes cell survival and invasion under metabolic stress by enhancing Warburg effect in pancreatic ductal adenocarcinoma. Dig Dis Sci. 2016;61(3):767-73.

14. Lincet $\mathrm{H}$, Icard $\mathrm{P}$. How do glycolytic enzymes favour cancer cell proliferation by nonmetabolic functions? Oncogene. 2015;34(29):3751-9.

15. Dong G, Mao Q, Xia W, Xu Y, Wang J, Xu L, et al. PKM2 and cancer: the function of PKM2 beyond glycolysis. Oncol Lett. 2016;11(3):1980-6.

16. Anastasiou D, Yu YM, Israelsen WJ, Jiang JK, Boxer MB, Hong BS, et al. Pyruvate kinase $\mathrm{M} 2$ activators promote tetramer formation and suppress tumorigenesis. Nat Chem Biol. 2012;8(10):839-47.

17. Iqbal MA, Gupta V, Gopinath P, Mazurek S, Bamezai RNK. Pyruvate kinase M2 and cancer: an updated assessment. FEBS Lett. 2014;588(16):2685-92.

18. Fang M, Shen Z, Huang S, Zhao L, Chen S, Mak TW, et al. The ER UDPase ENTPD5 promotes protein N-glycosylation, the Warburg effect, and proliferation in the PTEN pathway. Cell. 2010;143(5):711-24.

19. Israelsen WJ, Vander Heiden MG. ATP consumption promotes Cancer metabolism. Cell. 2010;143(5):669-71.

20. Giatromanolaki A, Sivridis E, Arelaki S, Koukourakis MI. Expression of enzymes related to glucose metabolism in non-small cell lung cancer and prognosis. Exp Lung Res. 2017;43(4-5):167-74.

21. Sanzey M, Rahim SAA, Oudin A, Dirkse A, Kaoma T, Vallar L, et al. Comprehensive Analysis of Glycolytic Enzymes as Therapeutic Targets in the Treatment of Glioblastoma. Plos One. 2015;10(5):e0123544.

22. Lee JH, Liu R, Li J, Wang YG, Tan L, Li XJ, et al. EGFR-Phosphorylated Platelet Isoform of Phosphofructokinase 1 Promotes PI3K Activation. Mol Cell. 2018; 70(2):197.

23. Cavalier MC, Kim SG, Neau D, Lee YH. Molecular basis of the fructose-2,6bisphosphatase reaction of PFKFB3: transition state and the C-terminal function. Proteins. 2012;80(4):1143-53.

24. Clem BF, O'Neal J, Tapolsky G, Clem AL, Imbert-Fernandez Y, Kerr DA, et al. Targeting 6-Phosphofructo-2-kinase ( PFKFB3) as a therapeutic strategy against Cancer. Mol Cancer Ther. 2013;12(8):1461-70.

25. Marsin AS, Bertrand L, Rider MH, Deprez J, Beauloye C, Vincent MF, et al. Phosphorylation and activation of heart PFK-2 by AMPK has a role in the stimulation of glycolysis during ischaemia. Curr Biol. 2000;10(20):1247-55.

26. Yalcin A, Clem BF, Imbert-Fernandez Y, Ozcan SC, Peker S, O'Neal J, et al. 6Phosphofructo-2-kinase (PFKFB3) promotes cell cycle progression and suppresses apoptosis via Cdk1-mediated phosphorylation of p27. Cell Death Dis. 2014;5(7):e1337. 
27. Kotiah SD, Caro J. Elevation of PFKFB3 and TIGAR levels in pancreatic cancer. J Clin Oncol. 2010;28(15):e14679-e14679.

28. Atsumi T, Chesney J, Metz C, Leng L, Donnelly S, Makita Z, et al. High expression of inducible 6-phosphofructo-2-kinase/fructose-2,6-bisphosphatase (iPFK-2; PFKFB3) in human cancers. Cancer Res. 2002;62(20):5881-7.

29. Li HM, Yang JG, Liu ZJ, Wang WM, Yu ZL, Ren JG, et al. Blockage of glycolysis by targeting PFKFB3 suppresses tumor growth and metastasis in head and neck squamous cell carcinoma. J Exp Clin Cancer Res. 2017;36. https://doi.org/10.1186/s13046-016-0481-1.

30. Zhu W, Ye L, Zhang JZ, Yu PF, Wang HB, Ye ZG, et al. PFK15, a Small Molecule Inhibitor of PFKFB3, Induces Cell Cycle Arrest, Apoptosis and Inhibits Invasion in Gastric Cancer. Plos One. 2016;11(9):e0163768.

31. Feng YH, Wu LS. mTOR up-regulation of PFKFB3 is essential for acute myeloid leukemia cell survival. Biochem Biophys Res Commun. 2017;483(2):897-903.

32. Clem BF, O'Neal J, Klarer AC, Telang S, Chesney J. Clinical Development of Cancer Therapeutics That Target Metabolism. QJM : Monthly Journal of the Association of Physicians. 2016;109(6):367-2.

33. Shi L, Pan H, Liu Z, Xie J, Han W. Roles of PFKFB3 in cancer. Signal transduction and targeted therapy. 2017;2:17044

34. Redman RA, et al. A phase I, dose-escalation, multi-center study of PFK-158 in patients with advanced solid malignancies explores a first-in-man inhbibitor of glycolysis. J Clin Oncol. 2015;33(15_suppl):TPS2606-TPS2606.

35. Badea L, Herlea V, Dima SO, Dumitrascu T, Popescu I. Combined gene expression analysis of whole-tissue and microdissected pancreatic ductal adenocarcinoma identifies genes specifically overexpressed in tumor epithelia. Hepato-Gastroenterology. 2008;55(88):2016-27.

36. Grynkiewicz G, Poenie M, Tsien RY. A new GENERATION of CA-2+ indicators with greatly improved fluorescence properties. J Biol Chem. 1985;260(6): 3440-50.

37. Goswami CP, Nakshatri H. PROGgeneV2: enhancements on the existing database. BMC Cancer. 2014;14:970.

38. Stratford JK, Bentrem DJ, Anderson JM, Fan C, Volmar KA, Marron JS, et al. A Six-Gene Signature Predicts Survival of Patients with Localized Pancreatic Ductal Adenocarcinoma. Plos Med. 2010;7(7):e1000307.

39. DiPietrantonio AM, Hsieh TC, Wu JM. Activation of caspase 3 in HL-60 cells exposed to hydrogen peroxide. Biochem Biophys Res Commun. 1999; 255(2):477-82.

40. Lu YF, Lei LN, Zhang YY, Liu RN, Xue XZ, Li L. Regulatory role and mechanism of NAC1 in cancer cell survival subjected to hypoxia. Int J Clin Exp Med. 2017;10(2):3155-61.

41. Daemen A, Peterson D, Sahu N, McCord R, Du XN, Liu BN, et al. Metabolite profiling stratifies pancreatic ductal adenocarcinomas into subtypes with distinct sensitivities to metabolic inhibitors. Proc Natl Acad Sci U S A. 2015; 112(32):E4410-E7.

42. Wang F, Liu HY, Hu LJ, Liu YF, Duan YJ, Cui R, et al. The Warburg effect in human pancreatic cancer cells triggers cachexia in athymic mice carrying the cancer cells. BMC Cancer. 2018;18:360.

43. Baek G, Tse YF, Hu ZP, Cox D, Buboltz N, McCue P, et al. MCT4 defines a glycolytic subtype of pancreatic Cancer with poor prognosis and unique metabolic dependencies. Cell Rep. 2014;9(6):2233-49.

44. Samad A, James A, Wong J, Mankad P, Whitehouse J, Patel W, et al. Insulin protects pancreatic Acinar cells from Palmitoleic acid-induced cellular injury. J Biol Chem. 2014;289(34):23582-95.

45. Mankad P, James A, Siriwardena AK, Elliott AC, Bruce JIE. Insulin protects pancreatic Acinar cells from cytosolic calcium overload and inhibition of plasma membrane calcium pump. J Biol Chem. 2012;287(3):1823-36.

46. Yue F, Cheng Y, Breschi A, Vierstra J, Wu WS, Ryba T, et al. A comparative encyclopedia of DNA elements in the mouse genome. Nature. 2014; 515(7527):355.

47. Low PS, Campanella EM, Chu HY. Regulation of glycolytic enzyme binding to the erythrocyte membrane. Blood. 2003;102(11):8A.

48. Hardin CD, Raeymaekers L, Paul RJ. Comparison of endogenous and exogenous sources of ATP in fueling Ca2+ uptake in smooth-muscle plasma-membrane vesicles. J Gen Physiol. 1992;99(1):21-40.

49. Webb BA, Dosey AM, Wittmann T, Kollman JM, Barber DL. The glycolytic enzyme phosphofructokinase-1 assembles into filaments. J Cell Biol. 2017; 216(8):2305-13.

50. Anderson RGW. The caveolae membrane system. Annu Rev Biochem. 1998; 67:199-225.

51. Brown DA, London E. Functions of lipid rafts in biological membranes. Annu Rev Cell Dev Biol. 1998;14:111-36.
52. El-Yazbi A, Cho WJ, Schulz R, Daniel E. Calcium extrusion by plasma membrane calcium pump is impaired in absence of intact caveolae. FASEB J. 2008;22(1_supplement):916.8-.8.

53. El-Yazbi AF, Cho WJ, Schulz R, Daniel EE. Calcium extrusion by plasma membrane calcium pump is impaired in caveolin-1 knockout mouse small intestine. Eur J Pharmacol. 2008;591(1-3):80-7.

54. Daniel EE, El-Yazbi A, Cho WJ. Caveolae and calcium handling, a review and a hypothesis. J Cell Mol Med. 2006;10(2):529-44.

55. Pang YH, Zhu H, Wu P, Chen JW. The characterization of plasma membrane Ca2+-ATPase in rich sphingomyelin-cholesterol domains. FEBS Lett. 2005; 579(11):2397-403.

56. Zhang J, Xiao P, Zhang X. Phosphatidylserine externalization in caveolae inhibits Ca2+ efflux through plasma membrane Ca2+-ATPase in ECV304. Cell Calcium. 2009;45(2):177-84.

57. Zakrzewicz D, Didiasova M, Zakrzewicz A, Hocke AC, Uhle F, Markart P, et al. The interaction of enolase-1 with caveolae-associated proteins regulates its subcellular localization. Biochem J. 2014;460:295-307.

58. Epstein T, Xu L, Gillies RJ, Gatenby RA. Separation of metabolic supply and demand: aerobic glycolysis as a normal physiological response to fluctuating energetic demands in the membrane. Cancer Metab. 2014;2:7.

59. Castro J, Ruminot I, Porras OH, Flores CM, Hermosilla T, Verdugo E, et al. ATP steal between cation pumps: a mechanism linking $\mathrm{Na}+$ influx to the onset of necrotic Ca2+ overload. Cell Death Differ. 2006;13(10):1675-85.

60. Caride AJ, Enyedi A, Penniston JT. Inhibition of the purified human red-cell CA-2+ pump by a monoclonal-antibody. Biochem J. 1989; 264(1):87-92.

61. Muallem S, Karlish SJD. Regulatory interaction between calmodulin and ATP on the red-cell CA2+ pump. Biochim Biophys Acta. 1980;597(3):631-6.

\section{Publisher's Note}

Springer Nature remains neutral with regard to jurisdictional claims in published maps and institutional affiliations.

\section{Ready to submit your research? Choose BMC and benefit from:}

- fast, convenient online submission

- thorough peer review by experienced researchers in your field

- rapid publication on acceptance

- support for research data, including large and complex data types

- gold Open Access which fosters wider collaboration and increased citations

- maximum visibility for your research: over $100 \mathrm{M}$ website views per year

At $\mathrm{BMC}$, research is always in progress.

Learn more biomedcentral.com/submissions 